\title{
Have the current and promising therapeutic options changed the surgeon's role in the treatment of pancreatic cancer?
}

\section{As atuais e promissoras opções terapêuticas modificaram o papel do cirurgião no tratamento do câncer de pâncreas?}

\author{
Emilio Vicente MD PhD FACS ${ }^{1}$, Yolanda Quijano Md PhD ${ }^{1}$.
}

$D$ ancreatic cancer (PC) has been a poor prognosis tumor for many years. The general factors that have always contributed to this, such as the anatomical location of the gland with its extensive lymphatic connections and the absence of clinical symptoms that allow an early diagnosis, add up to the scarce accomplishment of radical surgical procedures capable of achieving free-margins resections and the absence of effective chemo and radiotherapeutic procedures. Due to these circumstances, survival results have been very disappointing. Thus, a marked skepticism about this disease has been spread among patients and medical professionals ${ }^{1}$.

In the last ten years, notable therapeutic changes have occurred. Through them, it was possible to prove a slow but progressive improvement in results, with special repercussion in some reference groups that, due to their large series, have a wide experience in this field ${ }^{2}$.

The diagnostic methodology has improved remarkably. Throughradiology, endoscopyand radioisotopes, it is now possible to detect patients with disseminated and / or locally advanced disease. The greatest problem arises in the evaluation of vascular involvement. In our country, the combination of helical computed tomography and echo-endoscopy allows us to preoperatively detect 75\% of patients with PC who will require arterial or venous resection associated with pancreatic resection. The study of these patients should be as broad as possible to clearly define local commitment and its possible dissemination. The goal is to operate only those patients who may benefit from an oncologic resection, avoiding unnecessary procedures (surgeries with positive margins) that in some occasions surpass $40 \%$ of the operated patients ${ }^{3}$.
Venous infiltration of pancreatic tumors located in the cephalic position or on the isthmus was considered a criterion of irresectability for a long time. The technical complexity and the apparent small oncological benefit seemed to justify this attitude. Currently this criterion has been clearly overcome. A better surgical technique in the field of vascular surgery, achieved by specialists in pancreatic surgery, has made venous resection a standard procedure in the treatment of suchh patients. In order to obtain free margins, about $30 \%$ of our patients require venous resection.

From an oncological perspective, a question that currently does not have a clear answer is whether vascular invasion is always a sign of greater aggressiveness and, consequently, worse prognosis. In some patients it may be considered as such, especially in large lesions. However, in others, the aforementioned invasion stems only from an "unfavorable anatomical tumor location" and does not represent greater biological aggressiveness. Similar results obtained in many PC patients treated with or without vascular resection support the latter theory.

Arterial resection represents a different, though controversial, scenario. Virtually all clinical guidelines consider patients with this type of vascular involvement to be inoperable and unresectable. The first descriptions of combined pancreatic and arterial resections occurred in lesions located in the pancreatic body with invasion of the celiac trunk. The modified Appleby technique ${ }^{4}$, described in 1953, allows its realization without the need to perform hepatic revascularization. The hepatic vascularization is maintained through the pancreatoduodenal branches of the superior mesenteric artery. The

1 - Madrid Sanchinarro University Hospital, "Clara Campal" Oncology Center, Madrid, Spain. 
gastroduodenal artery must be preserved. The surgical challenge of this technique is the arterial resection, but the most complex part from the technical point of view is the subsequent reconstruction ${ }^{5}$.

Few groups in the world carry out resections of the common or proper hepatic artery, right hepatic artery or superior mesenteric artery in patients with PC. We established a rigorous selection criterion: nondisseminated lesions no larger than $3 \mathrm{~cm}$ and always after receiving a neoadjuvant treatment (radiochemical) for at least three months 6 .

On the other hand, vascular resection may in some occasions constitute an important technical necessity. Lymphadenectomy performed over arterial axes with intense perineural involvement may in some cases lead to arterial wall lesions, including rupture. The clinical manifestation is the presence of hemorrhage, in some cases massive, by the formation of arterial pseudoaneurysms. If occurring in the early postoperative days, this usually has a dramatic evolution. In those cases in which, after completion of lymphadenectomy, there are important changes in the artery characteristics, it should be resected ${ }^{7}$.

Of all the factors that contribute to increase PC patients'survival, the most important is the obtaining free margins. Therefore, vascular resection in selected patients can not and should not be a limiting factor to this goal, although this clearly increases the risk of the operation, especially if the resection is double, arterial and venous. Any PCpatient who will undergo surgery requires that the surgical team, in addition to trying to obtain an RO resection, have a sufficient degree of experience in vascular surgery to be able to perform it.

Another important aspect is to determine the real therapeutic benefit in these patients. It is difficult to establish it. Only the neoadjuvant treatment allows knowing the patient who, at least theoretically, can benefit. Patients with disease progression during or after neoadjuvant treatment should not be candidates for surgical treatment. Therefore, due to the difficulty in establishing adequate preoperative staging (perineural involvement), neoadjuvant treatment should be performed in all PCpatients.
In certain cases, other factorsinfluence patients' survival in an unknown way. Like other groups, we have described prolonged survival in patients with $\mathrm{PC}$, with or without multidisciplinary treatments. In general, these patients are carriers of small, well-differentiated tumors with no involvement of lymph nodes and / or resection margins. Some patients, however, had prolonged survival despite presenting with locally advanced tumors and poor prognostic factors, including metastases. These results demonstrate the heterogeneity of PCbiological behavior. In most cases, the biology of cancer, rather than the classic pathological factors, determinespatients' prognosis. But it is certain that there is an essential requirement to obtain a prolonged survival: the accomplishment of a radical surgery with free margins.

The new concepts on PC characteristics are opening new hopeful therapeutic perspectives. PC had a dense stroma. Pancreatic star cells (or myofibroblasts)play an important role in the formation and replacement of the stroma. This is not only a mechanical barrier, but is involved in the formation and progression to metastases. Stromal cells express a variety of proteins that are associated with resistance to treatment. These proteins represent new therapeutic targets. Therapy directed to the modification of the stroma allows to increase tumor vascularization, with consequent increased diffusion and, mainly, of the clinical efficacy of drugs on pancreatic tumors ${ }^{8,9}$.

In addition, within the tumors a subpopulation of neoplastic cells with pluripotential properties was identified. In PC these stem cells (1 to $5 \%$ of the tumor population) are resistant to radiation and chemotherapy, which could explain their inefficacy and the recent interest in directing treatment to these specific cells ${ }^{10}$.

New drugs include small molecules that inhibit signaling pathways and oncogenes. The recognition that both the tumor microenvironment and the neoplastic stem cells are critical elements of PC led to the development of agents, such as the inhibitors of the hedgehog signaling pathway, that block these components. The availability of preclinical models torecapitulate the complexity of this disease helps to establish strategies and priorities for the development of new drugs and innovative therapies. 
Ultimately, the PC genomic complexity demonstrates the heterogeneity of this type of cancer and advises individualization of treatment methods.

The role of the surgeon today is clearly defined in the treatment of PC: select patients who, according

\section{REFERENCES}

1. Hidalgo M. Pancreatic cancer. N Engl J Med. 2010;362(17):1605-17. Erratum in: N Engl J Med. 2010;363(3):298.

2. Garrido-Laguna I, Hidalgo M. Pancreatic cancer: from state-of-the-art treatment to promising novel therapies. Nat Rev Clin Oncol. 2015;12(6):319-34.

3. Vicente E, Quijano Y, lelpo B, Duran H, Diaz E, Fabra I, et al. is arterial infiltration still a criterion for unresectability in pancreatic adenocarcinoma? Cir Esp. 2014;92(5):305-15.

4. Appleby LH. The coeliac axis in the expansion of the operation for gastric carcinoma. Cancer. 1953;6(4):704-7.

5. Ielpo B, Ferri V, Caruso R, Duran H, Diaz E, Fabra I, et al. Alternative arterial reconstruction after extended pancreatectomy. Case report and some considerations of locally advanced pancreatic cancer. JOP. 2013;14(4):432-7.

6. Ielpo B, Duran H, Diaz E, Fabra I, Caruso R, Ferri V, et al. Preoperative treatment with gemcitabine plus nab- to their clinical situation and the characteristics of the disease, can benefit from the surgical treatment, and perform an R0 resection as safely as possible. This role has not changed in recent years, and it is still certain that there are more and more arguments to keep it.

paclitaxel is a safe and and effective chemotherapy for pancreatic adenocacinma. Eur J Surg Oncol. 2016;42(9):1394-400.

7. Ielpo B, Caruso R, Prestera A, De Luca GM, Duran $H$, Diaz $E$, et al. Arterial pseudoaneurysms following hepato-pancreato-biliary surgery: a single center experience. JOP. 2015;16(1):85-9.

8. Alvarez R, Musteanu M, Garcia-Garcia E, Lopez-Casas PP, Megias D, Guerra C, et al. Stromal disrupting effects of nab-paclitaxel in pancreatic cancer. $\mathrm{Br} J$ Cancer. 2013;109(4):926-33.

9. Alvarez R, Musteanu M, Garcia-Garcia E, Lopez-Casas PP, Megias D, Guerra C, et al. Reply: 'comments on stromal disrupting effects of nab-paclitaxel in pancreatic cancer'. Br J Cancer. 2014;111(8):1677-8.

10. Rubio-Viqueira $B$, Hidalgo M. Direct in vivo xenograft tumor model for predicting chemotherapeutic drug response in cancer patients. Clin Pharmacol Ther. 2009;85(2):217-21. 